MATEC Web of Conferences 52, 02007 (2016)

DOI: $10.1051 /$ matecconf/20165202007

(C) Owned by the authors, published by EDP Sciences, 2016

\title{
Adaptive Robotic Systems Design in University of Applied Sciences
}

\author{
Jos Gunsing, Fons Gijselhart, Nyke Hagemans, Hans Jonkers, Eric Kivits. Peter Klijn, Bart Kapteijns, \\ Diederich Kroeske, Hans Langen, Bart Oerlemans, Jan Oostindieand Joost van Stuijvenberg
}

Avans University of Applied Sciences, Centre of Expertise for Sustainable Innovation, Research Group for Robotics \& Mechatronics, Breda/'s-Hertogenbosch, The Netherlands

\begin{abstract}
In the industry for highly specialized machine building (small series with high variety and high complexity) and in healthcare a demand for adaptive robotics is rapidly coming up. Technically skilled people are not always available in sufficient numbers. A lot of know how with respect to the required technologies is available but successful adaptive robotic system designs are still rare. In our research at the university of applied sciences we incorporate new available technologies in our education courses by way of research projects; in these projects students will investigate the application possibilities of new technologies together with companies and teachers. Thus we are able to transfer knowledge to the students including an innovation oriented attitude and skills. Last years we developed several industrial binpicking applications for logistics and machining-factories with different types of 3D vision. Also force feedback gripping has been developed including slip sensing. Especially for healthcare robotics we developed a so-called twisted wire actuator, which is very compact in combination with an underactuated gripper, manufactured in one piece in polyurethane. We work both on modeling and testing the functions of these designs but we work also on complete demonstrator systems. Since the amount of disciplines involved in complex product and machine design increases rapidly we pay a lot of attention with respect to systems engineering methods. Apart from the classical engineering disciplines like mechanical, electrical, software and mechatronics engineering, especially for adaptive robotics more and more disciplines like industrial product design, communication \&multimedia design and of course physics and even art are to be involved depending on the specific application to be designed. Design tools like Vmodel, agile/scrum and design-approaches to obtain the best set of requirements are being implemented in the engineering studies from the early beginning.
\end{abstract}

\section{Introduction}

Avans University of Applied Sciences is situated in an industrialized part of the Netherlands.

In the technical field a shortage of highly skilled people is present. Thus production automation and robotics is an important theme. Many small and mediumsized companies are active in this industry notwith mass production but with building highly complex machines in small series. Also logistics/transport and material handling is important. Increasing logistic flexibility and a huge variety of products to be handled are typical trends. Apart from this in many types of industry a shortage of highly skilled technical people is present. Thus automation and robotics are important themes in 
combination with flexibility in capacity and the type of robot actions. With the industry in our region we agreed on focussing on robot gripper technology in combination with adaptive robotics.

Being a university of applied science we concentrate not so much on research into new technologies but we aim for making these new technologies applicable in new innovative products and systems. We do so in cooperation with universities and companies. Most of the projects we carry out in closecooperation with other universities of applied sciences like Fontys, Utrecht, Saxion and NHL. Most of the projects are funded by the universities of applied sciences themselves in combination withprojects funding (governmental organisation SIA ;Innovation Alliance Foundation)

The choice of the projects is the outcome of a process in which we take into account the business and product roadmaps of the involved companies and we combine these insights with very useful technology roadmap plus research agenda information from the organisation euRobotics[1, 2].

In the projects the research is being carried out with teachers, students and companies working together and all of them will increase their knowledge. Our students are our main workforces. The teachers are coaching them in the research process and go deep in the complex matters themselves. With this knowledge education and product innovation will be lifted to a higher level. Due to the increased system complexity and the increased multidisciplinarity we put also a lot of effort in education in systems engineering. We do aim for systems engineering as an umbrella under which many systematic design approaches can be applied like V-model, scrum/agile etc.

\section{Adaptive Robotic Systems Design}

\subsection{Systems setup}

Having chosen for gripper design as a focus subject we had to decide on which applications we should work for; In our case we choose for focus on industrial systems. Nevertheless we have had a chance to work on healthcare applications as well. We work on those types of healthcare gripper technologies, which are also applicable in industry for handling purposes.In the project roadmap for our research we aim for gradual increase in robot complexity especially in terms of robot/human cooperation. Based on a systematic approach in requirement analysis, choice of functions and modular design we have been working on several applications. More recently we have started with involvement of communication \& multimediadesign people in robot/human interaction. It is our strong opinion that they can help is to define the right functionality and to design a robot with which people are really happy. Also involvement of artists can help to magnify or even exaggerate robot functional aspects such that it helps people to come up with the right robot design ideas.

\subsubsection{Industrial applications}

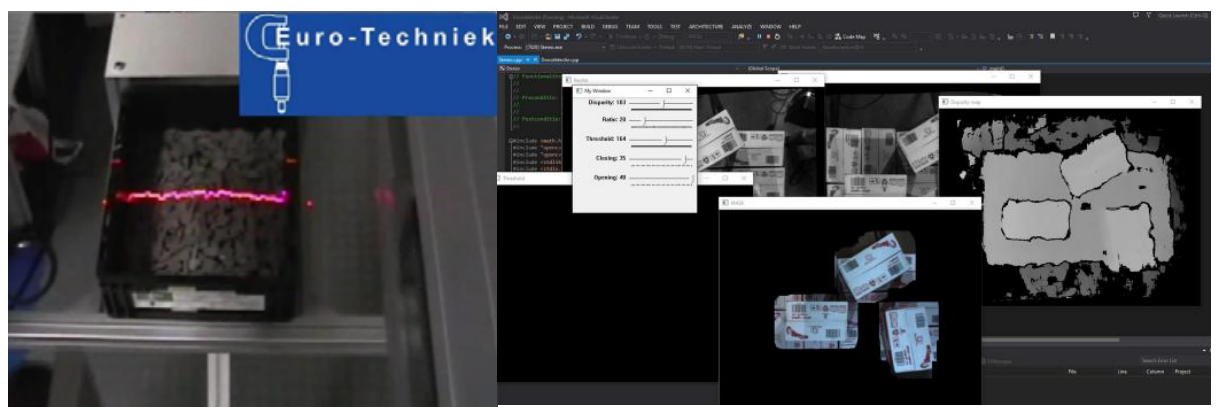

Figure 1.Binpicking application; scanningof non-oriented components in the bin. Box recognition and handling application: disparity map generation for box recognition; only upper layer of boxes should be visible. 
At this moment we have been working on binpicking applications for several years already. In our region many companies do work with metal or plastic components. Picking out the unsorted and nonoriented components out of a binis a challenging job especially when you are aiming for a very short learning time for the robot/vision/gripper system when trying to handle a new component. We are aiming now for learning times of one hour instead of weeks to obtain a robustly working system.

In a project in the near future we will be working on a robot-buddy for mechatronic systems assembly engineers. This engineer will teach his robot-buddy himself in order to take over part of the more routine-like jobs like putting in/screwing in bolts/nuts etcetera. Because he teaches this robotbuddy himself the buddy becomes more or less part of himself.

\subsubsection{Healthcare applications}

We are involved in a healthcare robotics project in which we develop a healthcare robot which is meant to help elderly people picking up objects which are on the floor or have fallen. We concentrated on the hand gripper function. Healthcare technology students studied the hand functions very thoroughly and came up with a set of user requirements includinguse cases, which are the basis for further development and engineering. During this process the healthcare technology department is still involved when reviewing the gripper design and especially when testing the gripper and robot system with the target user group.

\subsection{Technology focus}

In 2.1 we described the applications of the grippers we design. Several technologies will have to be a permanent subject of study. We apply several systems engineering design approaches over the whole process from user requirement/use case set up to concept generation/modelling, design, engineering and experimental validation including literature study in all those stages of the design process. We consider this to be the backbone of our educational program for esp. the mechatronics engineers but we aim to involve also other disciplines in the near future. Companies in our region struggle with the implementation of systems engineering but they are very much aware that it will help them to work successfully in multidisciplinary teams, to work together with other companies, to design modular product families, to change from a engineering-to-order to a configure-to-ordercompany.

Amongst others we base ourselves on the CAFCR-method [3], (Customer, Application, Functional, Concept, Realization views with respect to the product or system which must be developed) for obtaining the most suitable requirement/product concept combination.In gripper design you will find the complete set of mechatronic technologies you find in a robot (In the next chapter we will go into more detail with respect to these technologies):

- gripper design

- force and slip sensing plus control

- actuation/transmission

- 3D vision incl. data transfer At this moment we are gradually combining all the developed technologies in a complete gripper and robot system together with our cooperation partners in industry and (applied) sciences

\section{Technology}

\subsection{Gripping}

\subsubsection{Gripper system}

We have considered many alternatives as a gripper concept. It had to fulfil at least following requirements (in this case a healthcare robot for picking items from the floor): 


\section{MATEC Web of Conferences}

- Two finger gripper are sufficient for the required jobs/use cases

- Underactuated gripper design for cases of simplicity

- Easy manufacture/low product cost/easy exchangeability

The design, which proved to be the best proposition, has following features.

- All moving parts manufactured in one piece; waterjet cutting + milling from polyurethane;

-Simple design with use of elastic hinges with relatively low hysteresis

-External actuation possible; in this case with twisted cord plus electrical motor

- Moving parts covered with 3D printed covers

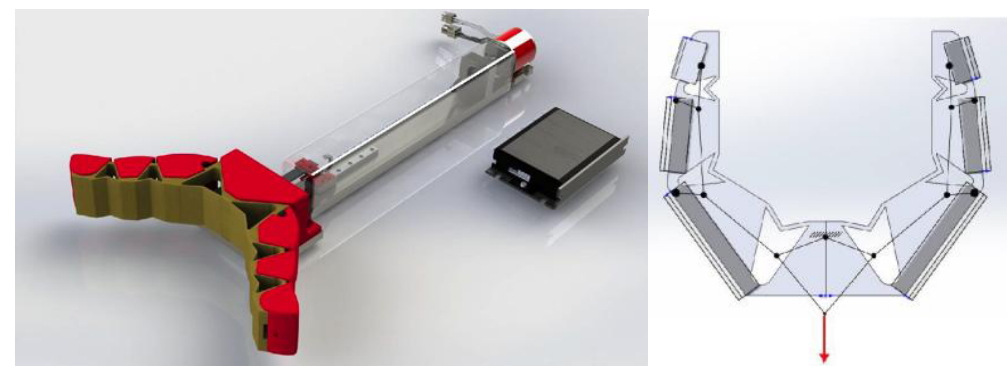

Figure 2.Complete gripper plus cross-sectional view of the underactuated gripper design with elastic hinges

At this moment only a limited amount of testwork has been carried out. First testresults are promising but extensive testwork including validation tests with potential users must be carried out.

\subsubsection{Force and slipsensing/-control}

Some time ago we started work on measuring and controlling the force in the gripper and we found out that it would be most appropriate also to measure and control slip when holding objects between the fingers. We use a slip-sensingconcept developed by the UEC Shimojo Laboratory (University of Tokyo). This slip sensor is based on a conductive rubber placed on top of an electrode. In pressurized condition the conductivity changes and a force-dependent signal can be measured. In [4] it is stated that just before slip occurs a high frequent signal occurs. At this moment we have a test rig up and running and the first promising indication of slip sensing i.e. high frequency signals have been seen. The test rig set up has been designed for very low hysteresis in the gripper finger force in both holding and slipping direction.

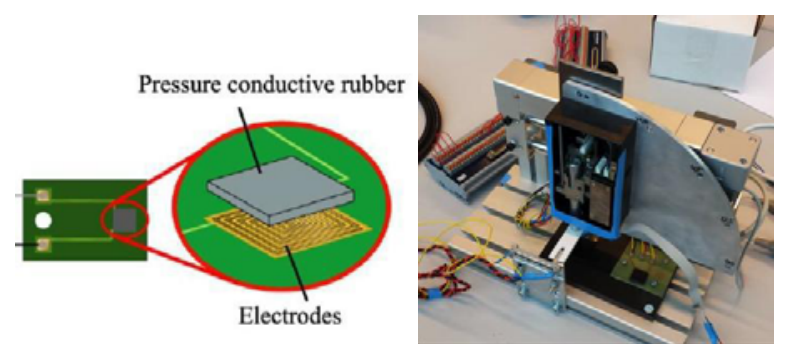

Figure 3.Force and slip sensitive sensor with pressure conductive rubber [4] andtestrig

Also a test gripper with very low friction/hysteresis has been designed. After evaluation of the results on this test rig we will be able to put the force and slip sensor on the gripper which was 
described in 3.1.1., which is much more robust but also has a higher hysteresis due to the polyurethane material choice.
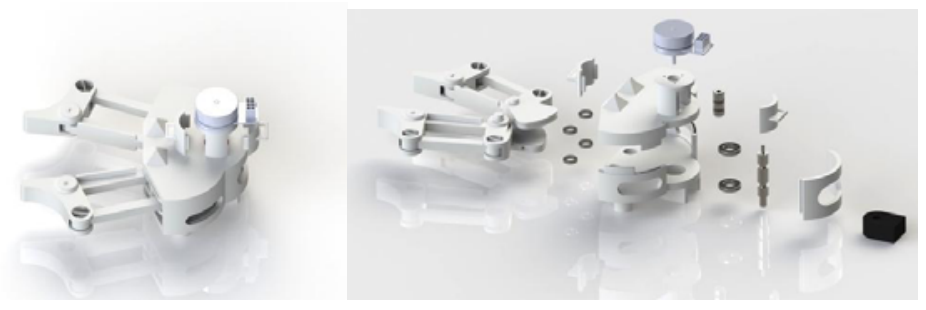

Figure 4.Low hysteresis gripper design for laboratory test; development and validation of force and slip sensor

\subsection{Actuation/transmission}

Our choice for a combination of a twisted cord and electrical motor has not been based on extensive studies but it appeared to be a actuator/transmission which was not quite known yet and papers were seldom at that time. A twisted cord consists of 2 or more cords twisted together; one end is fixed; the other end is directly attached to a motor shaft; when the motor turns the total length will become shorter and it can exercise a longitudinal force. This force can be applied to one or more gripper fingers. This actuator has its advantages when using it for moving the fingers in a gripper. The electrical motor can be placed in the arm of a robot at some distance keeping the gripper compact and the inertia low when moving the gripper together with the arm. A high torque motor is not necessary to move the fingers. In order to become more acquainted with the characteristics of a twisted cord we did set up a theoretical model and we also designed and built a test rig in order to predict and measure the relation between the force/torque and shortening of the twisted cord/number of motor revolutions.

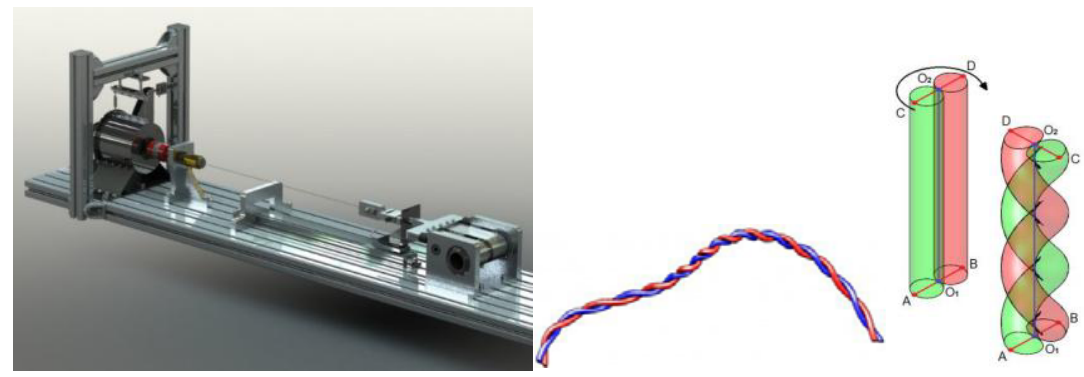

Figure 5.Twisted cord actuator/transmission; performance testrig, twisted cord and twisted cord model.

\subsection{Vision incl. data handling}

No robot and gripper system without a vision system ; since the early start of our robot investigations we have built up experience with vision systems based on several types of sensors;

- laser triangulation sensor

- time of flight camera

- stereovision(2 camera's)

We built up experience with these 3 types of vision systems; Also we have put a lot of effort in setting up our own FPGA based data handling system. This has been a big job and although we have obtained a lot of inside knowledge in this system we will now change to a purchased system and we 
will concentrate on the stereovisionsystems integration with the robot and gripper system including actuation force and slip sensing system. Data handling is also important; since robots tend to work (partially) autonomous we have several possibilities:

- data transformation to information is carried outdirectly on the robot preferably close to the camera

- data being sent over by Wi-Fi to operator dashboard or data storage depending on the application

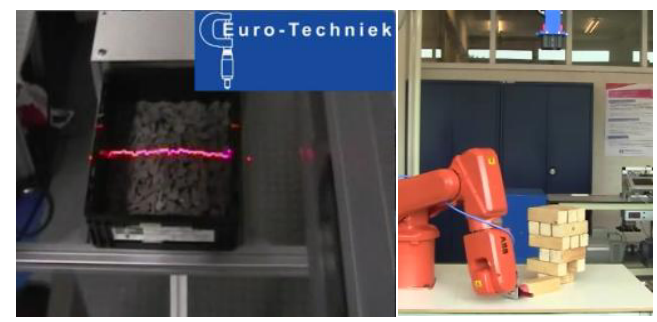

Figure 6.3D (2,5D) vision systems; laser triangulation and time of flight

\section{Conclusions \& Recommendations}

\subsection{Technical}

In about a year from now we will have working gripper $\&$ robot systems available. Meanwhile we will continue our research into increased levels of adaptive cooperative behaviour and of robots. Application of knowhow from artificial intelligence, behavioural sciences and communication \&multimedia design will help to further expand adaptive robotics applications.At this moment projects with these disciplines together with engineering are being set up as an eye opener for all those interested in robots and humans working more closely together.

\subsection{Educational}

Working with students in applied research helps them to develop an innovation-minded attitude while being very critical about their own way of working. Systems Engineering helps to line up process thinking and helps with tools for further development of their skills and mind.Meanwhile we incorporated research into the educational programs of the years 3 and 4 (4 year educational track)

\section{References}

1. Strategic Research Agenda For Robotics in Europe 2014-2020, SPARC Partnership for Robotics in Europe

2. Robotics 2020 Multi-Annual Roadmap for Robotics in Europe, Call 1 ICT23-Horizon 2020, Initial Release B 15/01/2014

3. G. Muller, A Multi View Method for Embedded Systems Architecting, Balancing Genericity and Specificity, thesis 2004, ISBN 90-5639-120-2, http://www.gaudisite.nl

4. S. Teshigawara,T. Tsutsumi,S. Shimizu, Y. Suzuki, A. Ming,M. Ishikawa, M. Shimojo, Highly Sensitive Sensor for Detection of Initial Slip and Its Application in a Multi-fingered Robot Hand, 2011 IEEE International Conference on Robotics and Automation pp.1097-102, 2011. 